\title{
Comparison of Metformin and Insulin in the Treatment of Gestational Diabetes: A Retrospective, Case-Control Study
}

\author{
Kristiina Tertti ${ }^{1}$, Ulla Ekblad ${ }^{1}$, Tero Vahlberg ${ }^{2}$ and Tapani Rönnemaa ${ }^{3}$ \\ ${ }^{1}$ Department of Obstetrics and Gynecology, Turku University Central Hospital, Turku, Finland. ${ }^{2}$ Department of Biostatistics, \\ University of Turku, Turku, Finland. ${ }^{3}$ Department of Internal Medicine, Turku University Central Hospital, Turku, Finland. \\ Address correspondence to: Kristiina Tertti,e-mail: kristiina.tertti@tyks.fi.
}

Manuscript submitted June 23, 2008; resubmitted August 23, 2008; accepted August 25, 2008

\section{Abstract}

OBJECTIVES: Limited data are available on metformin therapy in gestational diabetes. The aim of the study was to compare maternal and neonatal outcomes in patients with gestational diabetes mellitus (GDM) treated with metformin with those treated with insulin, or diet alone. STUDY DESIGN AND METHODS: We conducted a retrospective study that included 45 GDM women treated with metformin, 45 women treated with insulin and 83 women with no pharmacological treatment. Subjects were matched for pre-pregnancy body mass index (BMI) and age. RESULTS: There were no differences between the metformin-treated group and the other two groups in terms of maternal outcomes (total weight gain during pregnancy or after the diagnosis of GDM, pre-pregnancy hypertension, pregnancy induced hypertension, pre-eclampsia etc.). In the diagnostic 2hour oral glucose tolerance test, glucose values were slightly,

\section{Introduction}

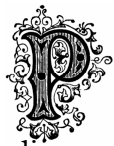

regnancy is a potentially glucose intolerant condition, and in all pregnancies, insulin sensitivity decreases as the pregnancy advances. This predisposes to the development of gestational diabetes mellitus (GDM), particularly in obese women with preexisting insulin resistance. GDM develops if there is inadequate insulin secretion to compensate for the increased insulin resistance [1]. but significantly, higher in the insulin group than in the metformin group $(p<0.003)$. Eighteen percent of mothers treated with metformin needed supplementary insulin therapy. No differences between the metformin-treated group and the other two groups (insulin, diet only) were observed in relation to mean birth weights, prevalence of macrosomia, or gestational weeks at delivery. The incidence of neonatal hypoglycemia was higher in the insulin group than in the metformin group $(p=0.03)$. There were no differences between the groups in other neonatal outcomes (small for gestational age, Apgar scores, umbilical artery $\mathrm{pH}$ or base excess, etc.). CONCLUSION: These retrospective data suggest that metformin is effective in controlling gestational diabetes and is not associated with a higher risk of maternal or neonatal complications compared with insulin.

Keywords: gestational diabetes • insulin ' metformin • OGTT $\cdot$ birth weight $\cdot$ hypoglycemia

GDM is diagnosed in approximately $3-7 \%$ of pregnancies $[2,3,4]$. The incidence of GDM increases in older and more obese pregnant women. GDM increases the risk of certain pregnancy complications like pregnancy-induced hypertension and adverse perinatal outcome, and carries the risk of later development of type 2 diabetes mellitus (T2DM). [1, 2, 4, 5].

Prospective randomized studies have recently demonstrated that effective treatment of hyperglycemia in women with GDM can reduce adverse perinatal out- 
comes [6]. The main purpose of treatment is to prevent fetal hyperinsulinemia and fetal macrosomia by reducing maternal glucose levels [7]. This is initially attempted by dietary and exercise counseling, but women often require additional treatment, which has traditionally been insulin [2]. The percentage of GDM patients needing pharmacological treatment varies from $20 \%$ to $60 \%$ in various studies [8]. However, the disadvantages of insulin for pregnant women, like other patients needing insulin, include the need to give injections, risk of hypoglycemia and risk of excessive weight gain $[8,9]$.

Theoretically, metformin is an alternative to insulin in the treatment of hyperglycemia during pregnancy. It decreases hepatic gluconeogenesis and improves peripheral glucose uptake [10]. It does not induce hypoglycemia and it is not associated with increased weight gain. Evidence supporting the use of metformin in pregnancy is available from studies in patients with polycystic ovary syndrome (PCOS). The syndrome is characterized by polycystic ovaries, oligo-amennorrhea, hyperandrogenism and insulin resistance. Metformin has been used in the treatment of infertility secondary to PCOS [4, 11]. Although it crosses the placenta, there is no evidence of adverse fetal effects $[12,13]$ or increased risk of major malformations when metformin is used in pregnant women [14]. Some PCOS studies, where metformin was used, reported a reduction in spontaneous abortion in the first trimester [15, 16] and metformin also seems to reduce the risk of GDM in PCOS-mothers [17]. Growth, motor and social development in the offspring of mothers who conceived and continued on metformin did not differ from that of control babies over the first 18 months of life [1].

In general, the number of studies reporting on the use of metformin in women with type 2 diabetes or GDM is still small. One retrospective study reported increased rates of pre-eclampsia and perinatal loss in a cohort of women with GDM or type 2 diabetes treated with metformin compared with a reference group treated with insulin [18],, but the groups were not well matched for age or body mass index. In the study of PCOS patients treated with metformin, the rate of preeclampsia did not differ compared with healthy control women even though women with PCOS were older and more obese [1]. There are two randomized studies comparing insulin and metformin in the treatment of GDM. One study with a small number of patients suggests that metformin is an effective alternative to insulin [19]. Another very recent multinational noninferiority study evaluated outcomes in terms of a composite of neonatal complications. It turned out that treatment with metformin fell within the preset condition of being less than $10 \%$ worse than with insulin [20].

As previous retrospective studies on the use of metformin in GDM were not properly controlled, and there is only one large prospective study on this subject, we performed a study in which the GDM patients with various treatments were matched for prepregnancy body mass index (BMI) and age. In this retrospective study, we compared GDM patients treated with metformin with those treated with insulin or with diet only. We evaluated maternal and neonatal outcomes of pregnancies to obtain additional data on whether metformin can be considered to be an alternative pharmacologic treatment to insulin in GDM.

\section{Materials and methods}

\section{Patients and measurements}

In Finland, the oral glucose tolerance test (OGTT) is performed in approximately $30 \%$ of all pregnant women, and approximately $5 \%$ of them need pharmacological treatment in addition to dietary therapy. In Turku University Central Hospital, metformin has been used as an alternative medication to insulin in GDM patients since 2002.

We conducted a retrospective analysis of all GDM pregnancies, where metformin was used from 20032006 at Turku University Central Hospital in South Western Finland. We included 45 metformin-treated women and the same number of insulin-treated GDM control patients. These patients were matched for prepregnancy body mass index (BMI, $\mathrm{kg} / \mathrm{m}^{2}$ ) and age. Matching for BMI was performed by stratifying the mothers into five different BMI categories $(<25,25-$ $\left.29,30-34,35-39, \geq 40 \mathrm{~kg} / \mathrm{m}^{2}\right)$. Mothers were agematched by stratifying them into three age categories $(<25,25-34$ and $\geq 35$ years). We also had a reference group of GDM patients treated with diet alone $(\mathrm{n}=$ 83). We aimed for a ratio of two diet alone patients to each metformin patient, although seven metformin patients had only one control patient treated with diet alone. Data were collected retrospectively from the maternal and neonatal patient records.

Only patients with singleton pregnancies were included. Most patients (158 out of 173) were Caucasians. All but 16 were Finnish by birth, one was of Russian origin, one Asian and 14 were Kurds.

All the women in this study had a 2 -hour $75 \mathrm{~g}$ OGTT at 11-32 weeks because they belonged to a 
high-risk group according to the protocol for the diagnosis of GDM used in Finland. The criteria for performing OGTT were those generally applied in Finland, i.e. one or more of the following criteria applied: BMI $\geq 25 \mathrm{~kg} / \mathrm{m}^{2}$, age $\geq 40$ years, previous macrosomic child, glucosuria during pregnancy, weight gain during pregnancy $\geq 20 \mathrm{~kg}$, previous GDM or suspected fetal macrosomia in the current pregnancy [21]. Criteria for GDM were at least two out of three abnormally high plasma glucose value measurements in the $75 \mathrm{~g}$ OGT'T (fasting $\geq 4.8,1 \mathrm{~h} \geq 10.0,2 \mathrm{~h} \geq 8.7$ $\mathrm{mmol} / \mathrm{l}$ ). All women visited the hospital for an interview and for dietary counseling on low glycemic index and low saturated fat eucaloric diet. They were taught self-monitoring of plasma glucose, with instructions to measure fasting and postprandial values at $90 \mathrm{~min}$ after main meals at least four times a day during three consecutive days. They were also given instructions to keep a dietary record and exercise diary on glucose measurement days.

The mode of treatment, based on self-monitored plasma glucose values, was determined within a week after starting monitoring. Pharmacologic treatment was started if fasting plasma glucose was $\geq 5.5 \mathrm{mmol} / 1$ at least twice, or fasting plasma glucose was at least once $\geq 5.5 \mathrm{mmol} / 1$ and postprandial value $\geq 7.8 \mathrm{mmol} / 1$, or fasting plasma glucose was $<5.5 \mathrm{mmol} / \mathrm{l}$ but postpran- dial value was at least twice $\geq 7.8 \mathrm{mmol} / \mathrm{l}$. If plasma glucose values remained below these limits, then dietary therapy was considered to be adequate.

The criteria for starting metformin were the same as for starting insulin. However, if fasting glucose value was above $7.0 \mathrm{mmol} / 1$ and/or postprandial values were above $10.0 \mathrm{mmol} / \mathrm{l}$, then insulin was always started. In 2003-2004, only one obstetrician specializing in diabetes and pregnancy used metformin or insulin, while other obstetricians used insulin in all GDM patients needing drug treatment. In 2005-2006, all obstetricians started using metformin or insulin. There were no strict criteria according to which one of the two drugs was started in those whose glucose values did not exceed the limits for compulsory insulin treatment, but approximately $20 \%$ of the women refused to start insulin injections. These women were treated with metformin.

All women receiving metformin gave their informed oral consent. The initial dose of oral metformin varied from $500 \mathrm{mg}$ once a day to $750 \mathrm{mg}$ twice a day. Insulin treatment was usually started with intermediate-acting insulin. For patients treated with metformin, insulin was subsequently used as supplementary treatment if required. All patients had a third trimester ultrasound evaluation of fetal weight. Patients treated with metformin or insulin were scheduled sev-

Table 1. Maternal data

\begin{tabular}{|c|c|c|c|c|c|c|c|c|}
\hline \multirow{2}{*}{$\begin{array}{l}\text { Parameter } \\
\text { Age (yr) }\end{array}$} & $\begin{array}{l}\text { Metformin } \\
(\mathrm{n}=45)\end{array}$ & $\begin{array}{l}\text { Insulin } \\
(\mathrm{n}=45)\end{array}$ & $\begin{array}{l}\text { Diet only } \\
(\mathrm{n}=83)\end{array}$ & \multirow{2}{*}{$\begin{array}{c}\begin{array}{c}\text { Overall } \\
\text { p-value }\end{array} \\
\text { NS }\end{array}$} & \multicolumn{2}{|c|}{$\begin{array}{l}\text { Metformin vs. insulin } \\
\text { p; OR }(95 \% \mathrm{CI})\end{array}$} & \multicolumn{2}{|c|}{$\begin{array}{l}\text { Metformin vs. diet only } \\
\text { p; OR }(95 \% \mathrm{CI})\end{array}$} \\
\hline & $32.8 \pm 5.0$ & $32.7 \pm 4.7$ & $32.4 \pm 5.3$ & & NS & & NS & \\
\hline BMI $\left(\mathrm{kg} / \mathrm{m}^{2}\right)$ & $34.0 \pm 6.4$ & $33.2 \pm 6.2$ & $33.7 \pm 6.2$ & NS & NS & & NS & \\
\hline Total weight gain (kg) & $10.2 \pm 6.7$ & $9.7 \pm 7.7$ & $8.8 \pm 4.8$ & NS & NS & & NS & \\
\hline Weight gain GDM $(\mathrm{kg})^{1}$ & $3.0 \pm 3.6$ & $3.5 \pm 5.2$ & $3.6 \pm 2.4$ & NS & NS & & NS & \\
\hline OGTT 0h (mmol/l) & $5.9 \pm 0.7$ & $6.3 \pm 0.8$ & $5.5 \pm 0.3$ & $<0.0001$ & 0.005 & & 0.000 & \\
\hline OGTT 1h (mmol/l) & $11.7 \pm 1.7$ & $12.7 \pm 2.0$ & $11.0 \pm 0.9$ & $<0.0001$ & 0.006 & & 0.03 & \\
\hline OGTT 2h (mmol/l) & $8.3 \pm 1.8$ & $9.5 \pm 2.2$ & $7.7 \pm 1.1$ & $<0.0001$ & 0.003 & & 0.07 & \\
\hline HbA1c at OGTT $(\%)$ & $5.7 \pm 0.4$ & $5.7 \pm 0.4$ & $5.5 \pm 0.3$ & 0.001 & NS & & 0.02 & \\
\hline Gestat. wk at OGTT & $24.8 \pm 5.5$ & $24.3 \pm 5.7$ & $27.1 \pm 2.4$ & 0.0002 & NS & & 0.004 & \\
\hline Gestat. wk at delivery & $38.4 \pm 1.4$ & $38.1 \pm 1.5$ & $38.9 \pm 2.0$ & 0.015 & NS & & NS & \\
\hline Primipara, n (\%) & $10(22.0)$ & $19(42.0)$ & $38(46.0)$ & 0.06 & 0.05 & $0.4(0.2-1.0)$ & 0.02 & $0.4(0.2-0.9)$ \\
\hline Smoking, n (\%) & 7 (15.6) & 7 (15.6) & $10(12.0)$ & NS & NS; & $1.0(0.3-3.0)$ & NS; & $1.4(0.5-3.7)$ \\
\hline $\mathrm{PPH}, \mathrm{n}(\%)$ & $2(4.4)$ & $4 \quad(8.9)$ & $2(2.4)$ & NS & NS; & $0.4(0.1-2.8)$ & NS; & $2.1(0.3-15.9)$ \\
\hline PIH, n (\%) & $0 \quad(0.0)$ & $1 \quad(2.2)$ & $3(3.6)$ & NS & & $0.0^{*}$ & & $0.0^{*}$ \\
\hline Pre-eclampsia, n (\%) & $4 \quad(8.9)$ & $4 \quad(8.9)$ & $2(2.4)$ & NS & NS; & $1.0(0.2-4.5)$ & NS; & $3.6(0.6-20.8)$ \\
\hline Induction of labor, $\mathrm{n}(\%)$ & $19(42.2)$ & $26(57.8)$ & $32(38.6)$ & NS & NS; & $0.5(0.2-1.2)$ & NS; & $1.2(0.6-2.6)$ \\
\hline
\end{tabular}

Legend: Data are mean \pm SD or number (n) and percent. BMI: body mass index. GDM: gestational diabetes mellitus. OGTT: oral glucose tolerance test. PPH: Pre-pregnancy hypertension. PIH: Pregnancy-induced hypertension. OR: odds ratio. CI: confidence interval. NS: not significant. ${ }^{1}$ Total weight gain after diagnosis of GDM. * OR could not be calculated because of zero cell values. 
eral visits to an obstetrician at the clinic and to a diabetologist, if necessary.

The following pregnancy and neonatal outcomes were recorded: pregnancy-induced hypertension $\mathrm{PIH}$; blood pressure elevation detected for the first time during pregnancy without proteinuria)[22], preeclampsia (increased blood pressure $>140 / 90 \mathrm{mmHg}$ accompanied by proteinuria $>0.3 \mathrm{~g} / 24 \mathrm{~h}$ )[22], birth weight (grams and SD for gestational weeks), macrosomia (birth weight $>4500 \mathrm{~g}$ and/or $>2 \mathrm{SD}$ ), the incidence of small for gestational age (SGA; birth weight $<2 \mathrm{SD}$ ), prematurity (birth $<37$ weeks of gestation), Apgar score at the age of $5 \mathrm{~min}$, umbilical artery $\mathrm{pH}<$ 7.05 and base excess, hypoglycemia (s-gluc $<2.6$ $\mathrm{mmol} / \mathrm{l}$, measured during the first two hours post partum), hyperbilirubinemia (need for phototherapy), need for intensive care treatment, respiratory distress syndrome (RDS), the mode of delivery (spontaneous, assisted or caesarean section) and shoulder dystocia.

The study was approved by the Turku University Central Hospital Ethics Committee.

\section{Statistical analysis}

A power calculation was performed for two end points, namely birth weight and incidence of hypoglycemia. A two-sided test with $80 \%$ power and significance level of 0.05 detected a $258 \mathrm{~g}$ mean difference between the groups of 45 metformin and 45 insulin patients. A two-sided test with $80 \%$ power and significance level of 0.05 found a $25.9 \%$ difference in hypoglycemia incidence between the metformin and the insulin-treated group.

Statistical analyses were performed using SAS software for Windows, version 9.1 (SAS Institute Inc., Cary, NC). The comparisons were made between all three groups (overall test) and pair-wise comparisons were made between mothers treated with metformin and insulin and mothers treated with metformin and diet. Continuous variables were compared between the groups using a linear mixed model, where pairmatching was used as a random effect. Dunnett's method was used for pair-wise comparisons. Condi-

Table 2. Neonatal data and mode of delivery

\begin{tabular}{|c|c|c|c|c|c|c|c|c|}
\hline \multirow{3}{*}{$\begin{array}{l}\text { Parameter } \\
\text { Birth weight (g) } \\
\text { Birth weight (SD-units) }\end{array}$} & \multirow{2}{*}{$\begin{array}{l}\text { Metformin } \\
(\mathrm{n}=45)\end{array}$} & \multirow{2}{*}{$\begin{array}{c}\begin{array}{c}\text { Insulin } \\
(\mathrm{n}=45)\end{array} \\
3759 \pm 642\end{array}$} & \multirow{2}{*}{$\begin{array}{c}\begin{array}{c}\text { Diet only } \\
(\mathrm{n}=83)\end{array} \\
3671 \pm 573\end{array}$} & \multirow{2}{*}{$\frac{\begin{array}{l}\text { Overall } \\
\text { p-value }\end{array}}{\text { NS }}$} & \multicolumn{2}{|c|}{$\begin{array}{l}\text { Metformin vs. insulin } \\
\quad \text { p; OR }(95 \% \mathrm{CI})\end{array}$} & \multicolumn{2}{|c|}{$\begin{array}{l}\text { Metformin vs. diet only } \\
\text { p; OR }(95 \% \mathrm{CI})\end{array}$} \\
\hline & & & & & NS & & NS & \\
\hline & $0.7 \pm 1.3$ & $0.9 \pm 1.6$ & $0.3 \pm 0.9$ & 0.08 & NS & & NS & \\
\hline Apgar score at $5 \mathrm{~min}$ & $8.6 \pm 0.8$ & $8.7 \pm 1.2$ & $8.9 \pm 0.6$ & NS & NS & & NS & \\
\hline $\mathrm{UA} \mathrm{pH}$ & $7.3 \pm 0.1$ & $7.3 \pm 0.1$ & $7.3 \pm 0.1$ & NS & NS & & NS & \\
\hline $\begin{array}{l}\text { UA base excess } \\
(\mathrm{mmol} / \mathrm{l})\end{array}$ & $-2.3 \pm 4.8$ & $-3.3 \pm 3.3$ & $-3.1 \pm 2.8$ & NS & NS & & NS & \\
\hline Neonate at NICU (days) & $2.4 \pm 8.1$ & $3.9 \pm 5.7$ & $2.8 \pm 7.0$ & NS & NS & & NS & \\
\hline Macrosomia, $\mathrm{n}(\%)^{1}$ & $7(15.6)$ & $10(22.2)$ & $10(12.0)$ & NS & NS; & $0.6(0.2-1.9)$ & NS & $1.3(0.5-3.6)$ \\
\hline SGA (<2 SD), n (\%) & $1 \quad(2.2)$ & $1 \quad(2.2)$ & 3 (3.6) & NS & NS; & $1.0(0.1-16.0)$ & NS; & $0.6(0.1-5.6)$ \\
\hline UA pH $<7.05, \mathrm{n}(\%)$ & $2(4.4)$ & $1 \quad(2.2)$ & 0 & NS & NS; & $2.0(0.2-22.1)$ & & $0.0^{*}$ \\
\hline $\begin{array}{l}\text { Neonates transferred to } \\
\text { NICU, n }(\%)\end{array}$ & $19(42.2)$ & $28(62.2)$ & $26(31.1)$ & 0.01 & 0.08 & $0.5(0.2-1.1)$ & NS; & $1.5(0.7-3.0)$ \\
\hline Hyperbil., n $(\%)^{2}$ & $12(26.7)$ & $11(24.4)$ & $17(20.5)$ & NS & NS; & $1.1(0.4-2.9)$ & NS; & $1.5(0.6-3.4)$ \\
\hline Hypoglycemia, n $(\%)^{3}$ & $15(34.1)$ & $26(57.8)$ & $16(19.3)$ & 0.0004 & 0.03 & $0.4(0.2-0.9)$ & 0.07 & $2.2(0.9-5.1)$ \\
\hline RDS, n (\%) & $\begin{array}{ll}0 & (0.0)\end{array}$ & $1 \quad(2.2)$ & $2(2.4)$ & NS & & $0.0^{*}$ & & $0.0^{*}$ \\
\hline Spontan. delivery, n (\%) & $27(60.0)$ & $30(66.7)$ & $62(74.7)$ & NS & NS; & $0.8(0.3-1.8)$ & NS; & $0.5(0.3-1.2)$ \\
\hline Assisted delivery, n (\%) & $5(11.1)$ & $5(11.1)$ & $6 \quad(7.2)$ & NS & NS; & $1.0(0.3-3.7)$ & NS; & $1.5(0.4-5.1)$ \\
\hline Caesarean section, $\mathrm{n}(\%)$ & $14(31.1)$ & $10(22.2)$ & $15(18.1)$ & NS & NS; & $1.6(0.6-4.2)$ & NS; & $2.2(0.9-5.2)$ \\
\hline Prematurity, n (\%) & $2(4.4)$ & $5(11.1)$ & $6 \quad(7.2)$ & NS & NS; & $0.4(0.1-2.0)$ & NS; & $0.6(0.1-2.9)$ \\
\hline
\end{tabular}

Legend: Data are mean \pm SD or number $(\mathrm{n})$ and percent. SD-units: indicate the birth weight deviation from the mean weight of newborn babies at given gestational weeks. UA: Umbilical artery. SGA: small for gestational age. NICU: neonatal intensive care unit. RDS: respiratory distress syndrome. OR: odds ratio. CI: confidence interval. NS: not significant. ${ }^{1}>2$ SD-units and/or $>4500$ g. ${ }^{2}$ Hyperbilirubinemia (need for phototherapy). ${ }^{3} \mathrm{n}=44$ in metformin group. ${ }^{*}$ OR could not be calculated because of zero cell values. 
tional logistic regression was performed to test the difference in dichotomous variables between the groups. Results were quantified using odds ratios (OR) with their 95\% confidence intervals (CI). We considered pvalues lower than 0.05 to be statistically significant.

\section{Results}

The metformin dose varied from $500 \mathrm{mg}$ to $2 \mathrm{~g}$ a day with a mean dose of $1 \mathrm{~g}$ a day. Eight out of $45 \mathrm{pa}-$ tients $(18 \%)$ required insulin in addition to metformin to maintain adequate glucose control (fasting glucose $<5.5 \mathrm{mmol} / \mathrm{l}$, postprandial glucose $<7.8 \mathrm{mmol} / \mathrm{l}$ ). In the insulin-treated group $(\mathrm{n}=45), 13$ patients were treated with short-acting insulin only (Humalog, Lilly, Indianapolis, USA; Actrapid or Novorapid, Novo Nordisk, Bægsevard, Denmark), 9 patients were treated with intermediate-acting insulin (Protaphane, Novo Nordisk) only, and 23 patients were treated with both short- and intermediate-acting insulin. Two patients in the metformin group were treated with oral corticosteroids in the first trimester of pregnancy, one because of asthma and the other because of idiopathic thrombocytopenia. Two patients in the metformin group and one patient in the insulin group had pregnancy-induced cholestasis in the third trimester of their pregnancy.

Table 1 shows maternal data. Glucose values (at 0 , $1,2 \mathrm{~h}$ ) in OGTT were significantly higher (respective p-values $0.005,0.006$ and 0.003 ) and the proportion of primiparas was slightly higher ( $p$-value 0.05$)$ in the insulin group than in the metformin group. Compared with the diet only group, fasting and 1-h glucose values in OGTT and HbA1c levels were significantly higher in the metformin group (respective p-values 0.0005, 0.03 and 0.02 ), whereas gestational weeks at OGTT and the proportion of primiparas were significantly lower (p-values 0.004 and 0.02). There were no statistically significant differences between the metformin and the other groups with respect to maternal age, prepregnancy BMI, total weight gain during pregnancy or after the diagnosis of GDM, smoking, pre-existence of hypertension, PIH or pre-eclampsia. Gestational weeks at birth and the incidence of induction of labor were also similar.

Table 2 shows the neonatal data. The incidence of neonatal hypoglycemia (s-gluc $<2.6 \mathrm{mmol} / \mathrm{l}$ ) treated with intravenous glucose was significantly higher in insulin-treated patients $(\mathrm{p}=0.03)$ compared with those treated with metformin (at our hospital, all the neonates born to diabetic mothers, including those with GDM, are tested for plasma glucose value during the first two hours post partum). There was no significant difference in the proportion of neonates sent to our neonatal intensive care unit (NICU) and no difference in the number of treatment days at NICU. There were also no statistically significant differences between the groups in relation to birth weight (grams or SD in gestational weeks), incidence of macrosomia or SGA, Apgar score at $5 \mathrm{~min}$, asphyxia (umbilical artery $\mathrm{pH}<$ 7.05), base excess in umbilical artery and neonatal hyperbilirubinemia needing phototherapy.

Three neonates had respiratory distress syndrome. All these neonates were born prematurely, at gestational weeks 30, 33 and 36, and none of their mothers belonged to the metformin group. No shoulder dystocia was detected in this study, but there were two clavicle fractures of the newborn, one in the metformin group and one in the insulin group, and one Erb's paresis in the insulin group. There were no differences between the groups in the proportion of mothers undergoing the different modes of delivery (spontaneous, assisted or caesarean section).

No significant differences were found in other neonatal outcome data between the metformin group and the diet only group and all findings were similar between the groups when patients treated with metformin in combination with insulin were excluded from the metformin group. There was one child in the metformin group, who had trisomy. This child died at the age of two months. No intrauterine deaths occurred. Thirteen patients gave birth prematurely $(<37$ weeks of gestational age): two in the metformin group (4.4\%, gestational ages 35 and 36 weeks), five in insulin group (11.1\%, gestational ages 34, 35 and 36 weeks), and six in the diet only group $(7.2 \%$, gestational ages 30 to 36 weeks).

\section{Discussion}

We found significantly fewer neonates with hypoglycemia during the first two hours post partum in the metformin-treated group compared with the insulintreated group $(p=0.03)$, whereas no difference in this respect was observed between the metformin and the diet only group. There was no significant difference in birth weight or neonate macrosomia between the three groups. Furthermore the rate of pre-eclampsia, caesarean section or neonatal RDS was similar in all three groups. These data are consistent with observations in PCOS patients treated with metformin [1, 23], but are inconsistent with the results obtained by Hellmuth et al. in a combined cohort of GDM and T2DM mothers [18]. In the latter study, increased rates of preeclampsia and perinatal loss were observed in mothers treated with metformin. 
A common problem in retrospective studies is that control groups are inadequately matched, as was the case in the study performed by Hellmuth et al. [18]. The metformin group had other increased risk factors for pre-eclampsia unrelated to metformin use, i.e. they were older and more obese. In addition, their antihyperglycemic medication was started seven weeks later than in the women treated with insulin. To our knowledge, our study is the first retrospective casecontrol study where control patients for metformin patients were matched for pre-pregnancy BMI and age. As a consequence, there were no significant differences in BMI or age of the patients between the groups. However, in the insulin group, the disturbance in glucose metabolism was slightly more severe according to the glucose values obtained in the diagnostic OGTT. This difference in maternal hyperglycemia, which may have also prevailed during delivery, may explain the higher rate of neonatal hypoglycemia in this group.

Until May 2008, only one small randomized study comparing metformin to insulin in GDM patients had been published [19]. In this study, patients' BMI, which may influence maternal and neonatal outcomes, differed significantly between the groups. Very recently, a larger prospective randomized study comparing metformin and insulin in the treatment of GDM was published. No differences were found in the composite endpoint of neonatal complications or birth weight, but the rate of preterm delivery was higher in metformin-treated mothers [20]. In our study, the incidence of preterm delivery was very low, but the study was not powered to detect differences in prematurity. In the study by Rowan et al., the rate of overall neonatal hypoglycemia was similar but the rate of severe hy-

\section{References}

1. Glueck C, Goldenberg N, Streicher P, Wang P. Metformin and gestational diabetes mellitus. Curr Diabet Rep 2003. 3:303312.

2. Kjos S, Buchanan T. Gestational diabetes mellitus. N Engl J Med 1999. 341(23):1749-1754.

3. Jovanovic L, Pettitt D. Gestational diabetes mellitus. JAMA 2001. 286:2516-2518.

4. Ben-Haroush A, Yogev Y, Hod M. Epidemiology of gestational diabetes mellitus and its association with type 2 diabetes. Diabet Med 2004. 21:103-113.

5. Kim C, Newton K, Knopp R. Gestational diabetes and the incidence of type 2 diabetes. Diabetes Care 2002. 25:1862-1868.

6. Crowther C, Hiller J, Moss J, McPhee A, Jeffries W, Robinson J. Effect of treatment of gestational diabetes mellitus on pregnancy outcomes. N Engl J Med 2005. 352:2477-2486.

7. Langer $\mathbf{O}$. Is normoglycemia the correct treshold to prevent complications in the pregnant diabetic patient? Diabet Rev 1996. poglycemia was lower in the metformin group compared to the insulin group [20]. Consistent with this result, the incidence of neonatal hypoglycemia was higher in insulin treated patients in our study. The caesarean section rate was not reported by Rowan et al. [20]. In our study, there was no difference in caesarean section rate between the metformin and the insulin group. The need for supplemental insulin was more frequent $(46 \%)$ in the study by Rowan et al. than in our study $(18 \%)$.

The limitation of our study lies in the selection and assignment of patients. Our study was not randomized and it is probable that insulin-treated patients were slightly more hyperglycemic than metformin-treated patients as judged by the glucose values in diagnostic OGTT. We had no strict criteria for assigning patients to metformin or insulin among those whose selfmonitored glucose values did not exceed the limits for compulsory insulin treatment (fasting glucose above $7.0 \mathrm{mmol} / 1$ or postprandial glucose above 10.0 $\mathrm{mmol} / \mathrm{l})$. The study had limited power to detect differences in many of the outcome variables, especially in those with low incidence.

\section{Conclusions}

We conclude that metformin appears to be effective in the treatment of GDM patients and does not seem to be associated with higher risks for maternal or neonatal complications compared with insulin. However, further sufficiently powered and randomized clinical studies are still needed, including long-term follow-up of children, in order to determine the role of metformin as an alternative treatment to insulin in GDM patients.
$4: 2-10$.

8. Langer $\mathbf{O}$. From educated guess to accepted practice: the use of oral antidiabetic agents in pregnancy. Clin Obst Gyn 2007. 4:959-971.

9. United Kingdom Prospective Diabetes Study Group (OKPDS) 13. Relative efficacy of randomly allocated diet, sulphonylurea, insulin, or metformin in patients with newly diagnosed non-insulin dependent diabetes followed for three years. BMJ 1995. 310(6972):83-88.

10. Kirpichinikov D, McFarlane S, Sowers J. Metformin: an update. Ann Intern Med 2002. 137:25-33.

11. McCarthy E, Walker S, McLachlan K, Boyle J, Permezel M. Metformin in obstetric and gynaecologic practice: a review. Obst Gyn Surv 2004. 59:118-127.

12. Nanovskaya T, Nekhayeva I, Partikeeva S, Hankins G, Ahmed M. Transfer of metformin across the dually perfused human placental lobule. Am J Obst Gyn 2006. 195:1081-1085.

13. Kovo M, Haroutiunian S, Feldman N, Hoffman A, Glezerman M. Determination of metformin transfer across the 
human placenta using a dually perfused ex vivo placental cotyledon model. Eur J Obst Gyn Repr Biol 2008. 136:29-33.

14. Gilbert C, Valois M, Koren G. Pregnancy outcome after forst-trimester exposure to metformin: a meta-analysis. Fertil Steril 2006. 86:658-663.

15. Glueck C, Phillips H, Cameron D, Sieve-Smith L, Wang P. Continuing metformin throughout pregnancy in women with polycystic ovary syndrome appears to safely reduce firsttrimester spontaneous abortion: a pilot study. Fertile Steril 2001. 75:46-52.

16. Jacubowicz D, Iuorno M, Jacubowicz S, Roberts K, Nestler J. Effects of metformin on early pregnancy loss in the polycystic ovary syndrome. J Clin Endocrinol Metab 2002. 87:524-529.

17. Glueck C, Wang P, Kobayashi S, Phillips H, Sieve-Smith L. Metformin therapy throughout pregnancy reduces the development of gestational diabetes in women with polycystic ovary syndrome. Fertil Steril 2002. 77:520-525.

18. Hellmuth E, Damm P, Molsted-Pedersen L. Oral hypogly- caemic agents in 118 diabetic pregnancies. Diabet Med 2000.17(7):507-511.

19. Moore L, Briery C, Clokey D, Martin R, Willford N, Bofill $\mathbf{J}$, Morrison J. Metformin and insulin in the management of gestational diabetes mellitus. J Reprod Med 2007. 52:1011-1015.

20. Rowan J, Hague W, Gao W, Battin M, Moore M, MiG Trial Investigators. Metformin versus insulin for the treatment of gestational diabetes. N Engl J Med 2008. 358:20032015.

21. Hyvönen K. Prevalence and screening for gestational diabetes. Doctoral Thesis, University of Kuopio, Finland, 1991.

22. Gifford R, August P, Cunningham G. Report of the national high blood pressure education program working group on high blood pressure in pregnancy. Am J Obst Gyn 2000. 183:S1-S22.

23. Glueck C, Bornovali S, Pranikoff J, Goldenberg N, Dharashivkar S, Wang P. Metformin, pre-eclampsia, and pregnancy outcomes in women with polycystic ovary syndrome. Diabet Med 2004. 21:829-836. 